\title{
Fabrication of an Aptamer-Coated Liposome Complex for the Detection and Profiling of Exosomes Based on Terminal Deoxynucleotidyl Transferase-Mediated
}

\section{Signal Amplification}

Lei Wang, ${ }^{\dagger}$ Yanhong Pan, ${ }^{\prime}$ Yunfei Liu, ${ }^{\dagger}$ Zhaowei Sun, ${ }^{\dagger}$ Yue Huang, ${ }^{\ddagger}$ Jinlong $\mathrm{Li}^{,}{ }^{\&}$ Jie Yang, ${ }^{\dagger}$ Yang Xiang ${ }^{\dagger}$ and Genxi Li ${ }^{*}{ }^{\dagger,}, \perp$

\footnotetext{
${ }^{\dagger}$ State Key Laboratory of Pharmaceutical Biotechnology, School of Life Sciences, Nanjing University, Nanjing 210023, P. R. China.

${ }^{\ddagger}$ Department of Food Science and Engineering, College of Light Industry and Food Engineering, Nanjing Forestry University, Nanjing 210037, China

\&Department of Laboratory Medicine, The Second Hospital of Nanjing, Nanjing University of Chinese Medicine, Nanjing 210003, P. R. China

${ }^{\perp}$ Center for Molecular Recognition and Biosensing, School of Life Sciences, Shanghai University, Shanghai 200444, P. R. China
}

\section{Corresponding Author}

*E-mail address: genxili@nju.edu.cn. 


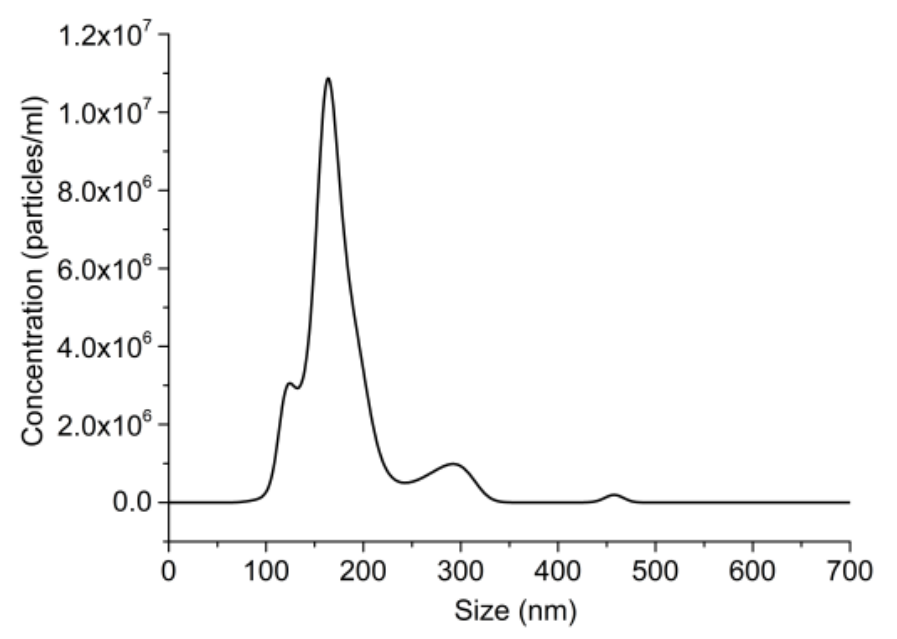

Figure S1. The size distribution characterization of MCF-7 derived exosomes using NanoSight. 
A

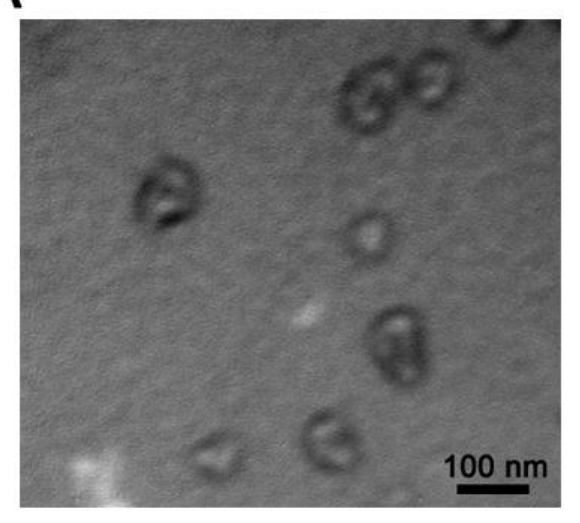

B

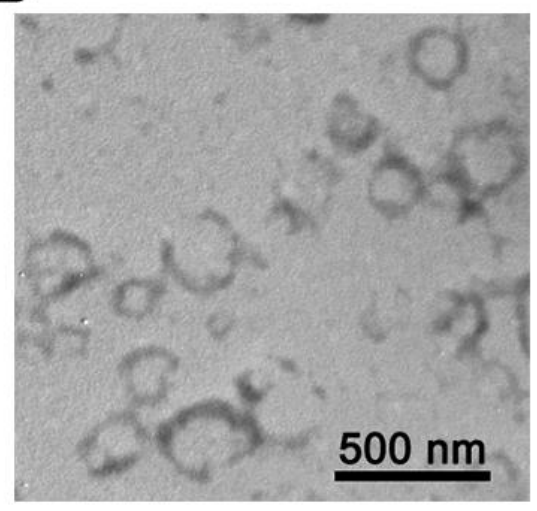

Figure S2. TEM images of exosomes (A) and DOTAP-liposomes (B). These samples are negatively stained using sodium phosphotungstate. 


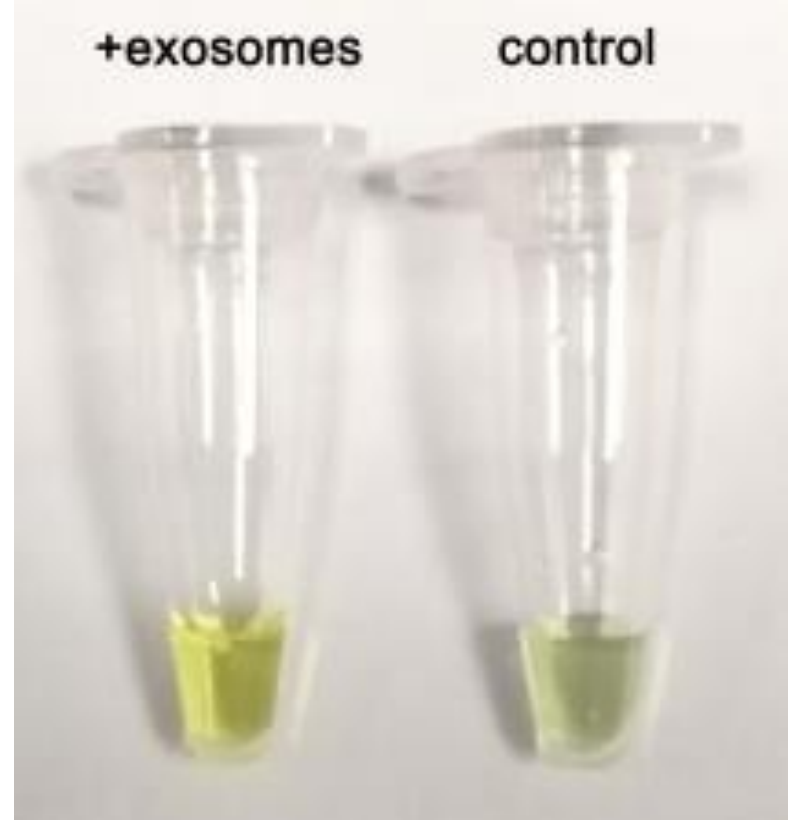

Figure S3. After the TdT-mediated polymerization, the colorimetric result comparison of the G-quadruplex + Hemin in the presence and absence of exosomes with the existence of $\mathrm{TMB}+\mathrm{H}_{2} \mathrm{O}_{2}$. The peroxidase reaction is terminated by adding $\mathrm{H}_{2} \mathrm{SO}_{4}$. 


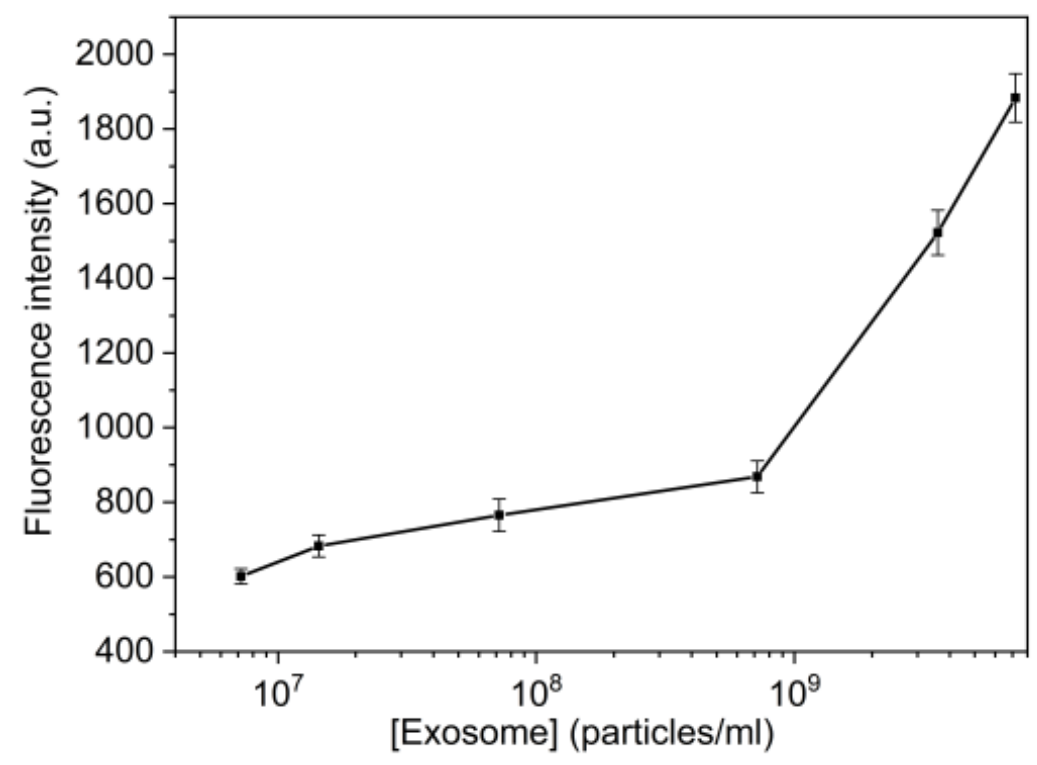

Figure S4. The exosomes detection performance of this strategy in DMEM medium with $1 \%$ $(\mathrm{v} / \mathrm{v})$ exosomes-free FBS. 
Table S1. The DNA sequence used in the study.

The overhang in the end of aptamers for better accessible to TdT is marked red.

\begin{tabular}{|l|l|}
\hline Target name & \multicolumn{1}{|c|}{ Sequence $\left(5^{\prime} \rightarrow 3^{\prime}\right)$} \\
\hline AptcD63 & CAC CCC ACC TCG CTC CCG TGA CAC TAA TGC TA TTT \\
\hline AptEpCAM & $\begin{array}{l}\text { CAC TAC AGA GGT TGC GTC TGT CCC ACG TTG TCA TGG } \\
\text { GGG GTT GGC CTG TTT }\end{array}$ \\
\hline AptmuC1 & GCA GTT GAT CCT TTG GAT ACC CTG G TTT \\
\hline AptheR2 & $\begin{array}{l}\text { GCA GCG GTG TGG GGG CAG CGG TGT GGG GGC AGC GGT } \\
\text { GTG GGG TTT }\end{array}$ \\
\hline Random DNA & $\begin{array}{l}\text { CCG TGT CTG GGG CCG ACC GGC GCA TTG GGT ACG TTG } \\
\text { TTG C }\end{array}$ \\
\hline
\end{tabular}


Table S2. Comparison with other assays methods for the detection of exosomes.

\begin{tabular}{ccccc}
\hline Method & $\begin{array}{c}\text { Detection limit } \\
(\text { particles/ } \mu \mathrm{L})\end{array}$ & $\begin{array}{c}\text { Washing } \\
\text { steps }\end{array}$ & $\begin{array}{c}\text { Signal } \\
\text { label }\end{array}$ & reference \\
\hline pH-responsive & $4.46 \times 10^{3}$ & yes & HRP & 1 \\
\hline dual-signal amplification & $1 \times 10^{2}$ & yes & $\begin{array}{c}\text { fluorescent } \\
\text { dye }\end{array}$ & 2 \\
\hline $\begin{array}{c}\text { zirconium-mediated } \\
\text { signal amplification }\end{array}$ & $7.6 \times 10^{3}$ & yes & label-free & 3 \\
\hline electrochemical detection & 200 & yes & HRP & 4 \\
\hline $\begin{array}{c}\text { paper-supported } \\
\text { aptasensor }\end{array}$ & $1.1 \times 10^{3}$ & yes & $\begin{array}{c}\text { gold } \\
\text { nanorods }\end{array}$ & 5 \\
\hline $\begin{array}{c}\text { magneto-electrochemical } \\
\text { detection }\end{array}$ & $3 \times 10^{3}$ & yes & HRP & 6 \\
\hline $\begin{array}{c}\text { electrochemical } \\
\text { ratiometric }\end{array}$ & 13 & yes & ferrocene & 7 \\
\hline $\begin{array}{c}\text { TdT-mediated signal } \\
\text { amplification }\end{array}$ & 360 & no & label-free & this work \\
\hline
\end{tabular}

\section{REFERENCES}

1. Yang, Y.; Li, C.; Shi, H.; Chen, T.; Wang, Z.; Li, G., A pH-Responsive Bioassay for Paper-Based Diagnosis of Exosomes Via Mussel-Inspired Surface Chemistry. Talanta 2019, 192, 325-330.

2. Huang, L.; Wang, D.-B.; Singh, N.; Yang, F.; Gu, N.; Zhang, X.-E., A dual-Signal Amplification Platform for Sensitive Fluorescence Biosensing of Leukemia-Derived Exosomes. Nanoscale 2018, 10 (43), 20289-20295.

3. Wang, L.; Yang, Y.; Liu, Y.; Ning, L.; Xiang, Y.; Li, G., Bridging Exosome and Liposome through Zirconium-Phosphate Coordination Chemistry: A New Method for Exosome Detection. Chem. Commun. 2019, 55 (18), 2708-2711.

4. Doldán, X.; Fagúndez, P.; Cayota, A.; Laíz, J.; Tosar, J. P., Electrochemical Sandwich Immunosensor for Determination of Exosomes Based on Surface Marker-Mediated Signal Amplification. Anal. Chem. 2016, 88 (21), 10466-10473.

5. Chen, X.; Lan, J.; Liu, Y.; Li, L.; Yan, L.; Xia, Y.; Wu, F.; Li, C.; Li, S.; Chen, J., A Paper-Supported Aptasensor Based on Upconversion Luminescence Resonance Energy Transfer for the Accessible Determination of Exosomes. Biosens. Bioelectron. 2018, 102, 582-588.

6. Jeong, S.; Park, J.; Pathania, D.; Castro, C. M.; Weissleder, R.; Lee, H., Integrated MagnetoElectrochemical Sensor for Exosome Analysis. ACS Nano 2016, 10 (2), 1802-1809.

7. Zhao, L.; Sun, R.; He, P.; Zhang, X., Ultrasensitive Detection of Exosomes by Target-Triggered Three-Dimensional DNA Walking Machine and Exonuclease III-Assisted Electrochemical Ratiometric Biosensing. Anal. Chem. 2019, 91 (22), 14773-14779. 